\title{
Income, inflammation and cancer mortality: a study of U.S. National Health and Nutrition Examination Survey mortality follow-up cohorts
}

\author{
Joshua E. Chan', Amandeep K. Mann², Daniel S. Kapp ${ }^{3}$ and David H. Rehkopf ${ }^{1 *}$ (D)
}

\begin{abstract}
Background: To estimate the relationship between inflammatory biomarkers and cancer mortality in a nationally representative sample of the U.S. population while controlling for education, occupation, and income.

Methods: Data were obtained from the U.S. National Health and Nutrition Examination Survey from 1988 to 1994 $(N=7817)$ and 1999-2002 $(N=2344)$. We fit Cox proportional hazard models to examine the relationship between C-reactive protein (CRP) and fibrinogen with cancer mortality.

Results: In the full Cox multivariate model, clinically raised CRP was associated with cancer mortality in NHANES 1988-1994 (>0.99 mg/dL: 95\%Cl: 1.04-2.13). However, across two inflammatory biomarkers (CRP and Fibrinogen), two NHANES time periods (1998-1994 and 1999-2002) and three income levels (12 strata in total), Hazard ratio confidence intervals did not include the null only for one association: CRP and cancer mortality among low income participants from 1988 to 1994 ( $H R=1.83,95 \%$ Cl: 1.10-3.04).

Conclusions: We find evidence that only in one unique stratum is earlier life CRP, and not fibrinogen, associated with prospective cancer mortality. After more complete control for socioeconomic confounding, CRP and fibrinogen do not predict cancer mortality in most subpopulations.
\end{abstract}

Keywords: Inflammation, C-reactive protein, Fibrinogen, Socioeconomic status, Cancer mortality

\section{Background}

Prior studies have found a relationship between higher levels of inflammation and worse cancer prognosis $[1,2]$. C-reactive protein (CRP) and fibrinogen are acute-phase proteins that are used to represent low-grade inflammation and associated with cancer incidence and mortality [3-5]. CRP has been correlated with an accumulation of myeloid derived cells

\footnotetext{
* Correspondence: drehkopf@stanford.edu

'Division of Primary Care and Population Health, Department of Medicine, Stanford University School of Medicine, 1701 Page Mill Road, Room 229, Palo Alto, Stanford, CA 94304, USA

Full list of author information is available at the end of the article
}

with suppressor functions, which are linked to several pathologies, and a heightened risk of cancer mortality, particularly in colorectal cancer [6, 7]. Furthermore, systemic inflammation, especially marked by high CRP levels, has been associated with higher morbidity and mortality among older ages $[8,9]$. Fibrinogen is another inflammatory marker that is associated with increased risk and poorer prognosis in epithelial ovarian and other cancers [10].

However, the impact of socioeconomic position (SEP) on these relationships is unclear. Socioeconomic position could act as a confounder or effect modifier of these relationships. One study found a $25 \%$ increase in

(c) The Author(s). 2020 Open Access This article is licensed under a Creative Commons Attribution 4.0 International License, which permits use, sharing, adaptation, distribution and reproduction in any medium or format, as long as you give appropriate credit to the original author(s) and the source, provide a link to the Creative Commons licence, and indicate if changes were made. The images or other third party material in this article are included in the article's Creative Commons licence, unless indicated otherwise in a credit line to the material. If material is not included in the article's Creative Commons licence and your intended use is not permitted by statutory regulation or exceeds the permitted use, you will need to obtain permission directly from the copyright holder. To view a copy of this licence, visit http://creativecommons.org/licenses/by/4.0/ The Creative Commons Public Domain Dedication waiver (http://creativecommons.org/publicdomain/zero/1.0/) applies to the data made available in this article, unless otherwise stated in a credit line to the data. 
CRP levels among participants who belonged to disadvantaged families during childhood [11]. In addition, Rachet et al. and Coleman et al. have found lower socioeconomic status to be associated with higher cancer mortality [12, 13]. Population-based studies have suggested a correlation between social behaviors and inflammation as risk factors for cancer and other chronic diseases [14]. Lower SEP and certain socio-behavioral patterns have been shown to be inversely correlated with CRP and fibrinogen, and higher systemic inflammation increases the risk of cancer diagnosis [14, 15]. Another report also found a relationship between lower SEP and worse cancer prognosis in melanoma patients [16]. The authors identified several confounders associated with melanoma survival; confounding variables included SEP, education level, and gender, all of which we measured in our study. However, there is limited information on the impact of inflammation on cancer prognosis adjusted for multiple measures of SEP. Previous publications lacked information on occupation, which has previously been evaluated as a predictor for socioeconomic status and life-space mobility [17-19]. Furthermore, previous reports lacked detailed information on demographics including race, body mass index, physical activity, and behavioral factors that could also act as confounders. For example, non-Hispanic blacks and Hispanic subgroups have been shown to have higher inflammatory marker levels compared to non-Hispanic whites [20]. Another study showed physical activity to be associated with improved survival across multiple cancers [21].

Given the limitations of these prior reports, we proposed to investigate whether 1 ) there is a meaningful relationship between inflammatory biomarkers and cancer mortality after controlling for multiple measures of SEP, and 2) whether family income acts as an effect modifier of the relationships between CRP and fibrinogen with cancer mortality. Finally, although our cohorts varied with respect to sample size, we proposed to determine whether temporal trends existed in the relationships between inflammatory biomarkers, socioeconomic status, and cancer mortality across two time periods.

\section{Methods}

\section{Data source}

Our study's population-based data are from the U.S. National Health and Nutrition Examination Survey (NHANES) from 1988 to 1994 (NHANES III) and 1999-2002 [22]. Conducted by the Centers for Disease Control and Prevention (CDC), NHANES examines the health and nutrition of individuals living in the U.S. by incorporating a multistage stratified, clustered probability sample of the U.S. civilian population. Data are collected through household interviews and in a mobile examination center (MEC). We use publicly available
NHANES data linked to the National Death Index (NDI) to assess cause-specific mortality.

\section{Inclusion/exclusion criteria}

From the full sample of NHANES III $(N=33,994)$ and NHANES 1999-2002 $(N=21,004)$, our data were limited due to NHANES collecting fibrinogen levels of participants aged 40 and over. After including participants from NHANES III $(N=8932)$ in this age group, we excluded those with prior history of cancer $(N=1045)$ and missing information on cancer history $(N=3)$. Participants with $\mathrm{CRP} \geq 10 \mathrm{mg} / \mathrm{dL}$ and above were also excluded $(N=8)$ because abnormally high CRP levels may be related to active infection rather than chronic inflammation [23]. Due to NHANES reporting all participants who are $\geq 90$ years old as "90," the participants listed as 90 years old and above were excluded $(N=86)$. The final NHANES III sample consisted of 7817 participants. Of the eligible participants in NHANES 1999-2002 $(N=2818)$, we excluded participants who reported pregnancy $(N=45)$, prior history of cancer $(N=334)$, had a missing history of cancer $(N=1)$, and had CRP levels $10 \mathrm{mg} / \mathrm{dL}$ and above $(N=4)$. In addition, we excluded participants $\geq 85$ years old who were reported as "85" in the NHANES 1999-2002 database $(N=124)$. The final NHANES 1999-2002 analytic sample was 2344 participants.

\section{Demographic and behavioral characteristics}

Participants were grouped as non-Hispanic white, nonHispanic black, or another race (includes Mexican/Hispanic, mixed race, and other race). Body mass index (BMI) was categorized as: underweight $\left(<18.5 \mathrm{~kg} / \mathrm{m}^{2}\right)$, normal $\left(18.5-24.9 \mathrm{~kg} / \mathrm{m}^{2}\right)$, overweight $\left(25.0-29.9 \mathrm{~kg} / \mathrm{m}^{2}\right)$, and obese $\left(\geq 30.0 \mathrm{~kg} / \mathrm{m}^{2}\right)$; physical activity as: lower, about the same, or higher compared to those of similar age based on physical activity questionnaire (PAQ); smoking as: current, former, and never.

\section{SEP indicators}

Participants' level of education was categorized as less than high school, high school, and above high school.

Poverty-income ratio (PIR), the ratio of family income to the poverty threshold, was used to define income. Our income categories are based on the U.S. Census defined income levels as poverty $($ ratio $<1)$, low income $(1.0 \leq$ ratio $<2.0)$, middle income $(2.0 \leq$ ratio $<4.0)$, and high income (ratio $\geq 4.0$ ) [24]. The "poverty" and "low income" groups were merged together in our analysis. We also created a measure of occupation based on the registrar general's class-based categorization with the categories of "Not Working", "White Collar and Professional", "White Collar, Semi-Routine", "Blue Collar, High Skill", and "Blue Collar, Semi-Routine" (Supplemental Table 1) [25]. The registrar general included labor force 
participants, classified as those who worked or were employed in a job or business within the last two weeks and excluding participants who solely performed housework.

\section{Inflammatory markers}

The serum inflammatory biomarker CRP was assessed by forming an antigen-antibody complex with latex particles coated with anti-CRP antibodies. A Behring Nephelometer measured light scattering after six minutes. CRP levels were then calculated using a calibration curve. The inflammatory biomarker fibrinogen levels were measured through thrombin clotting time [22]. In this assay, the enzyme thrombin was used to convert fibrinogen into its insoluble polymer, fibrin. In this study, CRP and fibrinogen levels were used as inflammatory biomarkers to evaluate their effects on cancer prognosis across income. The intermediate ranges for CRP are $0.22-0.99 \mathrm{mg} / \mathrm{dL}$ and 200 $400 \mathrm{mg} / \mathrm{dL}$ for fibrinogen [26-28].

In our sample, the mean CRP level was $0.45 \mathrm{mg} / \mathrm{dL}$ $(0.21-9.79 \mathrm{mg} / \mathrm{dL})$ in NHANES III and $0.45 \mathrm{mg} / \mathrm{dL}(0.01$ to $7.83 \mathrm{mg} / \mathrm{dL}$ ) in NHANES $1999-2002$. The mean fibrinogen level was $303 \mathrm{mg} / \mathrm{dL} \quad(19-957 \mathrm{mg} / \mathrm{dL})$ in NHANES III and $367 \mathrm{mg} / \mathrm{dL} \quad(120-808 \mathrm{mg} / \mathrm{dL})$ in NHANES 1999-2002. Due to the majority of the participants having CRP levels below the baseline detection level of $0.22 \mathrm{mg} / \mathrm{dL}$ and the NHANES labeling any value below $0.22 \mathrm{mg} / \mathrm{dL}$ as $0.21 \mathrm{mg} / \mathrm{dL}$, CRP was classified as a categorical variable. Using Swede et al. and Visser et al.'s classification methods, we categorized CRP levels of < $0.22 \mathrm{mg} / \mathrm{dL}, 0.22-0.99 \mathrm{mg} / \mathrm{dL}$, and $\geq 1.00 \mathrm{mg} / \mathrm{dL}$ as undetected, intermediate, and clinically raised CRP, respectively [26, 28]. Fibrinogen level was treated as a continuous variable in the test of association and multivariate analysis due to the normal distribution of participants' fibrinogen levels. However, for the survival curves, the intermediate range of fibrinogen was used as the cutoff [27].

\section{Measurement for mortality endpoint}

We obtained data on causes, month, and year of death from the National Center for Health Statistics (NCHS) linkage to the NDI, with follow-up through December 31, 2015 [22]. These data were linked to the NHANES dataset through probabilistic matching with social security number, birth date, and other personal data. The International Classification of Diseases codes for death were used to determine underlying causes of death for all cancer sites (C00-C97).

\section{Statistical analysis}

Strata, cluster, and weight variables were used to ensure that oversampling of any groups did not occur. For the NHANES III dataset, we used SDPSTRA6, SDPPSU6, and WTPFHX6 as strata, cluster, and weight variables, respectively. Similarly, with the NHANES 1999-2002 dataset, SDMVSTRA, SDMVPSU, and WTMEC4YR were used as strata, cluster, and weight variables for analysis use. Using the appropriate Chi-square and t-tests, the associations with income levels were examined. Additionally, we analyzed the associations of demographic, socioeconomic, and behavioral characteristics in our study with CRP and fibrinogen levels (Supplemental Table 2) (Supplemental Table 3). We created unadjusted Kaplan-Meier curves to investigate the differences in cancer-specific survival in the overall income level groups and inflammation. Furthermore, income level groups were stratified to examine how the difference in survival of participants varied by CRP and fibrinogen level. Age was used as a baseline for time to follow-up, death from other causes or cancer death [29]. Additionally, we utilized Cox-proportional hazard models to adjust for potential confounders of CRP and fibrinogen levels for cancer mortality. Due to the strong correlation between CRP and fibrinogen (NHANES III: $\mathrm{B}=0.003$, $p<0.001$; NHANES 1999-2002: $\mathrm{B}=0.004, p<0.001$ ) and the uncertain nature of their causal ordering, separate multivariable analyses were performed for these inflammatory markers. Cox-proportional hazard models were fit to test for cancer mortality associations for each variable, represented as Model 1 in Supplemental Table 4. Furthermore, 3 models were fit based on a priori specification for demographic (race and gender), socioeconomic (income, education, and occupation), and behavioral (BMI, physical activity, and smoking status) factors. A Directed Acyclic Graph (DAG) was constructed to describe the possible confounders in the association between the inflammatory biomarkers and cancer mortality (Supplemental Fig. 1).

Proportional hazard assumptions were assessed for all covariates in the full model. Since NHANES III and NHANES 1999-2002 were separately grouped by strata, cluster, and weights, the two datasets were analyzed separately. We examined the association of CRP and fibrinogen levels with cancer mortality in the overall multivariate analysis. In the subset analyses, the Coxproportional hazard model was stratified by income levels and gender to investigate the association between inflammation and cancer mortality in each income group. Data analyses were conducted using $\mathrm{SAS}^{\circ} 9.3$ (SAS Institute Inc., Cary, NC, USA). This study was exempted from the IRB approval because it is a public use data file and does not contain identifying information of participants.

\section{Results}

Of 7817 participants (median age: 53 years; range: $40-$ 89) in the NHANES III sample, $79.0 \%$ were white, $9.8 \%$ 
were black, and $11.1 \%$ were of another race. The sample consists of $52.4 \%$ females and $47.6 \%$ males. About $61.6 \%$ of participants had education levels of high school or below, while $38.4 \%$ of participants had education levels above high school. Low, middle, and high-income levels were represented in $43.8,38.6$, and $17.7 \%$ of participants, respectively (Table 1 ). The mean follow-up time for low, middle and high-income levels was 7.9 years, 7.8 years, and 7.4 years ranging between 3.3 and 9 years.

Of 2344 participants (median age: 52 years; range: $40-$ 84) in the NHANES 1999-2002 sample, $74.5 \%$ were white, $10.6 \%$ were black, and $14.8 \%$ were of another race. $51.6 \%$ of participants were female, and $48.4 \%$ male. Roughly $49.5 \%$ of participants had education levels of high school or below, whereas $50.4 \%$ of participants were educated above high school. Low, middle, and highincome levels were represented in 38.6, 35.4, and 26.0\% of participants, respectively (Table 2). The mean followup time for low, middle and high-income levels was 7.7 years, 7.4 years, and 7.3 years ranging between 3.3 and 8.3 years.

In the NHANES III dataset, lower income was associated with older age $(p<0.001)$, black or other non-white participants $(p<0.001)$, and those with education levels of high school or below $(p<0.001)$. (Table 1$)$.

Similarly, for participants in the NHANES 1999-2002 dataset, those in the lower income cohort were older $(p<0.001)$, black or non-white $(\mathrm{p}<0.001)$, and educated at or below the high school level $(p<0.001)$.

We found an inverse relationship between income levels and both CRP and fibrinogen levels in both time periods. For the low, middle, and high income levels in NHANES III, the mean CRP levels were $0.57 \mathrm{mg} / \mathrm{dL}$, $0.43 \mathrm{mg} / \mathrm{dL}$, and $0.38 \mathrm{mg} / \mathrm{dL}(\mathrm{p}<0.001)$, and the mean fibrinogen levels were $319 \mathrm{mg} / \mathrm{dL}, 298 \mathrm{mg} / \mathrm{dL}$, and 294 $\mathrm{mg} / \mathrm{dL}$, respectively $(\mathrm{p}<0.001)$ (Table 1$)$. For the low, middle, and high income levels in NHANES 1999-2002, the mean CRP levels were $0.52 \mathrm{mg} / \mathrm{dL}, 0.46 \mathrm{mg} / \mathrm{dL}$, and $0.39 \mathrm{mg} / \mathrm{dL}(p=0.003)$, and the mean fibrinogen levels were $385 \mathrm{mg} / \mathrm{dL}, 371 \mathrm{mg} / \mathrm{dL}$, and $351 \mathrm{mg} / \mathrm{dL}$, respectively $(p=0.003)$ (Table 2$)$.

We found no statistically significant relationship between income levels and cancer mortality (Supplemental Fig. 2). We examined the relationship between inflammatory markers and cancer mortality and found that those with higher CRP levels had a significantly higher risk of cancer mortality in the NHANES III dataset (log-rank $p<0.001)$, yet there was no significant association between CRP and cancer mortality in the NHANES 1999-2002 dataset (logrank $p=0.10$ ) (Supplemental Fig. 3). Similarly, higher fibrinogen levels were correlated to higher risk of cancer mortality in the NHANES III dataset (log-rank $p=0.03)$, but the correlation was not statistically significant in the NHANES 1999-2002 dataset (log-rank $p=0.28$ ) (Supplemental Fig. 4).
In the full Cox multivariate model, clinically raised CRP was associated with cancer mortality in the NHAN ES III (>0.99 mg/dL: 95\%CI: 1.04-2.13) (Table 3). The relationship between CRP and cancer mortality was not significant in the NHANES 1999-2002 cohort, regardless of socioeconomic status $(0.22-0.99 \mathrm{mg} / \mathrm{dL}$ : $95 \% \mathrm{CI}$ : 0.55-1.59) (>0.99 mg/dL: 95\%CI: 0.92-2.42) (Table 3). Fibrinogen was also not associated with cancer mortality in both the NHANES III (95\%CI: 1.000-1.002) and NHANES 1999-2002 (95\%CI: 0.998-1.002) samples (Table 4).

In the multivariate analysis evaluating CRP, being female was associated with lower cancer mortality compared to being male in both the NHANES III (HR = 0.68, 95\%CI: $0.54-0.87 ; p=0.003)$ and the NHANES 1999-2002 (HR $=0.52, \quad 95 \% \mathrm{CI}: \quad 0.27-0.99 ; \quad p=0.047)$ datasets (Table 3 ). In addition, in the analysis evaluating fibrinogen, being female was associated with lower cancer mortality in the NHANES III $(\mathrm{HR}=0.70,95 \% \mathrm{CI}$ : $0.54-0.89 ; p=0.005$ ) (Table 4). We further stratified the full Cox multivariate model by income groups to assess for associations of each income group with inflammation levels and cancer mortality. After adjusting for all factors, we found that higher CRP levels were associated with lower cancer survival among low income participants in the NHANES III dataset $(0.22-0.99 \mathrm{mg} / \mathrm{dL}$ : $\mathrm{HR}=1.23,95 \% \mathrm{CI}: \quad 0.88-1.74 ; p=0.22) \quad(>0.99 \mathrm{mg} / \mathrm{dL}:$ $\mathrm{HR}=1.83$, 95\%CI: $1.10-3.04 ; p=0.02$ ), but the relationship was not significant in NHANES 1999-2002 (Table 5, Fig. 1, Supplemental Fig. 5).

\section{Discussion}

In this study of 10,161 participants without a cancer diagnosis at baseline, we found that lower income was associated with increased biomarkers of inflammation. After controlling for measures of socioeconomic position, we found associations between CRP and cancer mortality in the NHANES III sample, but we found non-significant trends in the NHANES 1999-2002 sample.

Our findings that higher CRP and was associated with higher cancer mortality compared to their respective undetected and intermediate levels, but also that undetected and intermediate inflammation levels were associated with similar cancer survival rates, may indicate that having above-average inflammation levels is more closely associated with higher cancer mortality than their respective below-average levels. In addition, it indicates the greater importance that anti-inflammatory drugs may have in lowering cancer mortality perhaps in subgroups with low income and high C-reactive protein levels. Nevertheless, our findings that this only occurred in 1 out of 12 strata of the population caution that this may not be of relevance to current cohorts. 
Table 1 Demographics, Socioeconomic Status, and Behavioral Characteristics by Income Levels from NHANES III (1988-1994)

\begin{tabular}{|c|c|c|c|c|c|}
\hline Characteristics & Total $(N=7817)$ & Low Income $(N=3420)$ & Middle Income $(N=3014)$ & High Income $(N=1383)$ & $P$-value \\
\hline Age (years) & & & & & $<0.001^{\mathrm{a}}$ \\
\hline Median (Range) & $53(40-89)$ & $58(40-89)$ & $53(40-89)$ & $51(40-89)$ & \\
\hline Younger than $55 \mathrm{yrs}$ & $51.6 \%$ & $40.7 \%$ & $52.0 \%$ & $61.0 \%$ & \\
\hline 55 yrs. or older & $48.4 \%$ & $59.3 \%$ & $48.0 \%$ & $39.0 \%$ & \\
\hline Race/Ethnicity & & & & & $<0.001^{\mathrm{a}}$ \\
\hline White & $79.0 \%$ & $64.1 \%$ & $80.5 \%$ & $90.5 \%$ & \\
\hline Black & $9.8 \%$ & $17.7 \%$ & $8.7 \%$ & $4.2 \%$ & \\
\hline Hispanics/Other & $11.1 \%$ & $18.2 \%$ & $10.7 \%$ & $5.3 \%$ & \\
\hline Gender & & & & & $<0.001^{\mathrm{a}}$ \\
\hline Male & $47.6 \%$ & $42.3 \%$ & $47.3 \%$ & $52.8 \%$ & \\
\hline Female & $52.4 \%$ & $57.7 \%$ & $52.7 \%$ & $47.2 \%$ & \\
\hline Education & & & & & $<0.001^{\mathrm{a}}$ \\
\hline Below High School & $29.1 \%$ & $55.6 \%$ & $29.6 \%$ & $14.8 \%$ & \\
\hline High School/Equivalent & $32.5 \%$ & $27.1 \%$ & $36.6 \%$ & $26.2 \%$ & \\
\hline Above High School & $38.4 \%$ & $7.9 \%$ & $29.2 \%$ & $62.9 \%$ & \\
\hline Occupation & & & & & $<0.001^{a}$ \\
\hline Not Working & $2.8 \%$ & $5.3 \%$ & $2.4 \%$ & $1.1 \%$ & \\
\hline White Collar and Professional & $26.7 \%$ & $11.7 \%$ & $23.5 \%$ & $45.0 \%$ & \\
\hline White Collar, Semi-Routine & $22.8 \%$ & $15.6 \%$ & $23.7 \%$ & $27.9 \%$ & \\
\hline Blue Collar, High Skill & $13.3 \%$ & $14.3 \%$ & $15.1 \%$ & $9.6 \%$ & \\
\hline Blue Collar, Semi-Routine & $34.5 \%$ & $53.1 \%$ & $35.3 \%$ & $16.4 \%$ & \\
\hline Body Mass Index $\left(\mathrm{kg} / \mathrm{m}^{2}\right)$ & & & & & $0.001^{\mathrm{a}}$ \\
\hline Median (Range) & $26.6(11.7-67.3)$ & $27.3(13.3-60.0)$ & $26.5(11.7-67.3)$ & $25.9(15.9-55.9)$ & \\
\hline Underweight & $1.6 \%$ & $2.2 \%$ & $1.5 \%$ & $1.0 \%$ & \\
\hline Normal & $34.5 \%$ & $31.3 \%$ & $35.0 \%$ & $36.6 \%$ & \\
\hline Overweight & $37.1 \%$ & $34.3 \%$ & $37.2 \%$ & $39.5 \%$ & \\
\hline Obese & $26.9 \%$ & $32.2 \%$ & $26.4 \%$ & $22.8 \%$ & \\
\hline Smoking & & & & & $<0.001^{\mathrm{a}}$ \\
\hline Never & $42.2 \%$ & $41.8 \%$ & $43.2 \%$ & $41.0 \%$ & \\
\hline Former & $33.9 \%$ & $28.9 \%$ & $33.4 \%$ & $39.3 \%$ & \\
\hline Current & $23.9 \%$ & $29.4 \%$ & $23.4 \%$ & $19.7 \%$ & \\
\hline Physical Activity ${ }^{b}$ & & & & & $<0.001^{\mathrm{a}}$ \\
\hline More Active & $37.7 \%$ & $28.4 \%$ & $37.9 \%$ & $45.8 \%$ & \\
\hline Less Active & $21.6 \%$ & $27.8 \%$ & $20.5 \%$ & $17.5 \%$ & \\
\hline About Same & $40.7 \%$ & $43.8 \%$ & $41.6 \%$ & $36.7 \%$ & \\
\hline C-Reactive Protein & & & & & $<0.001^{\mathrm{a}}$ \\
\hline Mean (Range) & $0.45(0.21-9.79)$ & $0.57(0.21-9.79)$ & $0.43(0.21-8.90)$ & $0.38(0.21-9.20)$ & \\
\hline$<0.22 \mathrm{mg} / \mathrm{dL}$ & $66.1 \%$ & $56.0 \%$ & $67.9 \%$ & $72.8 \%$ & \\
\hline $0.22-0.99 \mathrm{mg} / \mathrm{dL}$ & $24.7 \%$ & $30.7 \%$ & $23.7 \%$ & $20.7 \%$ & \\
\hline$>0.99 \mathrm{mg} / \mathrm{dL}$ & $9.2 \%$ & $13.3 \%$ & $8.4 \%$ & $6.5 \%$ & \\
\hline Fibrinogen & & & & & $<0.001^{c}$ \\
\hline Mean (Range) & 303 (19-957) & 319 (30-928) & 298 (19-957) & $294(85-806)$ & \\
\hline
\end{tabular}

${ }^{a}$ Chi-Square tests were performed for categorical variables

${ }^{b}$ Physical activity compared to others of the same age

c $\mathrm{t}$-tests were performed for the continuous variables 
Table 2 Demographics, Socioeconomic Status, and Behavioral Characteristics by Income Levels from NHANES $1999-2002$

\begin{tabular}{|c|c|c|c|c|c|}
\hline Characteristics & Total $(N=2344)$ & Low Income $(N=904)$ & Middle Income $(N=830)$ & High Income $(N=610)$ & $P$-value \\
\hline Age (years) & & & & & $<0.001^{\mathrm{a}}$ \\
\hline Median (Range) & $52(40-84)$ & $55(40-84)$ & $53(40-84)$ & $51(40-84)$ & \\
\hline Younger than $55 \mathrm{yrs}$ & $55.5 \%$ & $48.5 \%$ & $51.7 \%$ & $64.0 \%$ & \\
\hline 55 yrs. or older & $44.5 \%$ & $51.5 \%$ & $48.3 \%$ & $36.0 \%$ & \\
\hline Race/Ethnicity & & & & & $<0.001^{a}$ \\
\hline White & $74.5 \%$ & $60.5 \%$ & $71.7 \%$ & $87.3 \%$ & \\
\hline Black & $10.6 \%$ & $14.9 \%$ & $12.5 \%$ & $5.9 \%$ & \\
\hline Hispanics/Other & $14.8 \%$ & $24.6 \%$ & $15.8 \%$ & $6.8 \%$ & \\
\hline Gender & & & & & $0.03^{\mathrm{a}}$ \\
\hline Male & $48.4 \%$ & $43.7 \%$ & $50.1 \%$ & $50.4 \%$ & \\
\hline Female & $51.6 \%$ & $56.3 \%$ & $49.9 \%$ & $49.6 \%$ & \\
\hline Education & & & & & $<0.001^{a}$ \\
\hline Below High School & $24.3 \%$ & $46.2 \%$ & $24.3 \%$ & $8.5 \%$ & \\
\hline High School/Equivalent & $25.2 \%$ & $27.8 \%$ & $28.0 \%$ & $20.8 \%$ & \\
\hline Above High School & $50.4 \%$ & $26.0 \%$ & $47.7 \%$ & $70.7 \%$ & \\
\hline Occupation & & & & & $<0.001^{\mathrm{a}}$ \\
\hline Not Working & $2.1 \%$ & $4.8 \%$ & $2.2 \%$ & $0.2 \%$ & \\
\hline White Collar and Professional & $32.1 \%$ & $17.1 \%$ & $25.4 \%$ & $49.1 \%$ & \\
\hline White Collar, Semi-Routine & $23.6 \%$ & $17.4 \%$ & $24.6 \%$ & $27.2 \%$ & \\
\hline Blue Collar, High Skill & $13.4 \%$ & $15.5 \%$ & $15.4 \%$ & $10.0 \%$ & \\
\hline Blue Collar, Semi-Routine & $28.8 \%$ & $45.1 \%$ & $32.3 \%$ & $13.5 \%$ & \\
\hline Body Mass Index $\left(\mathrm{kg} / \mathrm{m}^{2}\right)$ & & & & & $0.64^{\mathrm{a}}$ \\
\hline Median (Range) & $27.7(12.0-66.4)$ & $28.1(15.8-66.4)$ & $27.9(12.0-63.9)$ & $27.4(16.5-61.2)$ & \\
\hline Underweight & $1.1 \%$ & $1.5 \%$ & $1.1 \%$ & $0.9 \%$ & \\
\hline Normal & $27.7 \%$ & $26.8 \%$ & $26.8 \%$ & $29.1 \%$ & \\
\hline Overweight & $37.4 \%$ & $34.7 \%$ & $39.4 \%$ & $37.5 \%$ & \\
\hline Obese & $33.8 \%$ & $37.0 \%$ & $32.6 \%$ & $32.5 \%$ & \\
\hline Smoking & & & & & $0.002^{\mathrm{a}}$ \\
\hline Never & $45.5 \%$ & $44.4 \%$ & $43.1 \%$ & $48.5 \%$ & \\
\hline Former & $31.7 \%$ & $26.2 \%$ & $34.2 \%$ & $33.5 \%$ & \\
\hline Current & $22.8 \%$ & $29.4 \%$ & $22.7 \%$ & $18.0 \%$ & \\
\hline Physical Activity $^{b}$ & & & & & $0.001^{a}$ \\
\hline More Active & $40.6 \%$ & $36.0 \%$ & $44.7 \%$ & $40.2 \%$ & \\
\hline Less Active & $20.8 \%$ & $27.1 \%$ & $19.3 \%$ & $17.7 \%$ & \\
\hline About Same & $38.6 \%$ & $37.0 \%$ & $36.0 \%$ & $42.1 \%$ & \\
\hline C-Reactive Protein & & & & & $<0.001^{a}$ \\
\hline Mean (Range) & $0.45(0.01-7.83)$ & $0.52(0.01-7.83)$ & $0.46(0.01-6.90)$ & $0.39(0.01-6.80)$ & \\
\hline$<0.22 \mathrm{mg} / \mathrm{dL}$ & $44.9 \%$ & $38.9 \%$ & $43.7 \%$ & $50.5 \%$ & \\
\hline $0.22-0.99 \mathrm{mg} / \mathrm{dL}$ & $44.2 \%$ & $45.5 \%$ & $45.3 \%$ & $42.2 \%$ & \\
\hline$>0.99 \mathrm{mg} / \mathrm{dL}$ & $10.9 \%$ & $15.5 \%$ & $11.1 \%$ & $7.3 \%$ & \\
\hline Fibrinogen & & & & & $0.003^{c}$ \\
\hline Mean (Range) & 367 (120-808) & 385 (173-808) & 371 (177-747) & 351 (120-715) & \\
\hline
\end{tabular}

${ }^{a}$ Chi-Square tests were performed for categorical variables

${ }^{b}$ Physical activity compared to others of the same age

${ }^{c}$ t-tests were performed for the continuous variables 
Table 3 Multivariate Cox Proportional Hazard Model Adjusted for CRP

\begin{tabular}{|c|c|c|c|c|c|c|}
\hline \multirow[t]{2}{*}{ Characteristics } & \multicolumn{3}{|c|}{ NHANES III 1988-1994 } & \multicolumn{3}{|c|}{ NHANES 1999-2002 } \\
\hline & Hazard Ratio & 95\% Confidence Interval & P-value & Hazard Ratio & 95\% Confidence Interval & $P$-value \\
\hline \multicolumn{7}{|l|}{ C-Reactive Protein (mg/dL) } \\
\hline$<0.22 \mathrm{mg} / \mathrm{dL}^{\mathrm{a}}$ & 1 & & & 1 & & \\
\hline $0.22-0.99 \mathrm{mg} / \mathrm{dL}$ & 1.01 & $0.81-1.26$ & 0.90 & 0.94 & $0.55-1.59$ & 0.80 \\
\hline$>0.99 \mathrm{mg} / \mathrm{dL}$ & 1.49 & $1.04-2.13$ & 0.03 & 1.49 & $0.92-2.42$ & 0.10 \\
\hline \multicolumn{7}{|l|}{ Race/Ethnicity } \\
\hline White ${ }^{a}$ & 1 & & & 1 & & \\
\hline Black & 1.25 & $0.97-1.61$ & 0.09 & 1.29 & $0.75-2.23$ & 0.35 \\
\hline Hispanics/Other & 0.90 & $0.61-1.34$ & 0.61 & 1.30 & $0.64-2.64$ & 0.46 \\
\hline \multicolumn{7}{|l|}{ Gender } \\
\hline Male $^{a}$ & 1 & & & 1 & & \\
\hline Female & 0.68 & $0.54-0.87$ & 0.003 & 0.52 & $0.27-0.99$ & 0.047 \\
\hline \multicolumn{7}{|l|}{ Education } \\
\hline Below High School ${ }^{\mathrm{a}}$ & 1 & & & 1 & & \\
\hline High School/Equivalent & 1.31 & $0.99-1.72$ & 0.06 & 1.19 & $0.57-2.52$ & 0.63 \\
\hline Above High School & 1.17 & $0.79-1.74$ & 0.41 & 0.73 & $0.31-1.68$ & 0.44 \\
\hline \multicolumn{7}{|l|}{ Income Level } \\
\hline Low $^{a}$ & 1 & & & 1 & & \\
\hline Middle & 0.85 & $0.70-1.05$ & 0.12 & 1.20 & $0.70-2.05$ & 0.49 \\
\hline High & 0.79 & $0.56-1.11$ & 0.17 & 1.58 & $0.78-3.19$ & 0.20 \\
\hline \multicolumn{7}{|l|}{ Occupation } \\
\hline Blue Collar, Semi-Routine ${ }^{a}$ & 1 & & & 1 & & \\
\hline White Collar and Professional & 0.89 & $0.60-1.32$ & 0.54 & 0.73 & $0.40-1.31$ & 0.28 \\
\hline White Collar, Semi-Routine & 0.94 & $0.60-1.46$ & 0.76 & 1.04 & $0.57-1.91$ & 0.89 \\
\hline Blue Collar, High Skill & 1.05 & $0.67-1.62$ & 0.84 & 1.31 & $0.88-1.97$ & 0.18 \\
\hline Not Working & 1.34 & $0.78-2.30$ & 0.28 & 0.91 & $0.26-3.11$ & 0.87 \\
\hline \multicolumn{7}{|l|}{ Body Mass Index $\left(\mathrm{kg} / \mathrm{m}^{2}\right)$} \\
\hline Underweight $^{a}$ & 1 & & & 1 & & \\
\hline Normal & 1.40 & $0.73-2.67$ & 0.31 & 1.71 & $0.33-8.86$ & 0.51 \\
\hline Overweight & 1.10 & $0.61-1.99$ & 0.74 & 1.91 & $0.41-8.81$ & 0.40 \\
\hline Obese & 1.64 & $0.84-3.17$ & 0.14 & 2.58 & $0.52-12.72$ & 0.24 \\
\hline \multicolumn{7}{|l|}{ Smoking } \\
\hline Never $^{a}$ & 1 & & & 1 & & \\
\hline Former & 1.67 & $1.15-2.42$ & 0.01 & 2.23 & $1.32-3.77$ & 0.004 \\
\hline Current & 3.86 & $2.87-5.18$ & $<0.001$ & 6.99 & $4.11-11.88$ & $<0.001$ \\
\hline \multicolumn{7}{|l|}{ Physical Activity ${ }^{b}$} \\
\hline About the Same ${ }^{a}$ & 1 & & & 1 & & \\
\hline Less Active & 1.15 & $0.86-1.53$ & 0.35 & 1.15 & $0.57-2.32$ & 0.68 \\
\hline More Active & 0.69 & $0.55-0.85$ & 0.001 & 0.87 & $0.47-1.62$ & 0.65 \\
\hline
\end{tabular}

${ }^{a}$ Reference group

${ }^{b}$ Physical activity compared to others of same age

Prior studies have also shown lower income to be associated with inflammation, specifically with elevated levels of CRP and fibrinogen. Nazmi and colleagues performed a systematic review of 32 studies and found that poverty and non-white race were correlated with higher CRP levels [30]. Others found similar results in a crosssectional study surveyed in the U.S., with higher inflammation in low income participants adjusted for 
Table 4 Multivariate Cox Proportional Hazard Model Adjusted for Fibrinogen

\begin{tabular}{|c|c|c|c|c|c|c|}
\hline \multirow[t]{2}{*}{ Characteristics } & \multicolumn{3}{|c|}{ NHANES III 1988-1994 } & \multicolumn{3}{|c|}{ NHANES 1999-2002 } \\
\hline & Hazard Ratio & 95\% Confidence Interval & $P$-value & Hazard Ratio & 95\% Confidence Interval & $P$-value \\
\hline Fibrinogen (mg/dL) & 1.001 & $1.000-1.002$ & 0.07 & 1.00 & $0.998-1.002$ & 0.75 \\
\hline \multicolumn{7}{|l|}{ Race/Ethnicity } \\
\hline White ${ }^{a}$ & 1 & & & 1 & & \\
\hline Black & 1.26 & $0.98-1.63$ & 0.07 & 1.30 & $0.74-2.27$ & 0.35 \\
\hline Hispanics/Other & 0.89 & $0.60-1.32$ & 0.56 & 1.27 & $0.62-2.58$ & 0.50 \\
\hline \multicolumn{7}{|l|}{ Gender } \\
\hline Male $^{a}$ & 1 & & & 1 & & \\
\hline Female & 0.70 & $0.54-0.89$ & 0.005 & 0.52 & $0.27-1.01$ & 0.05 \\
\hline \multicolumn{7}{|l|}{ Education } \\
\hline Below High School ${ }^{a}$ & 1 & & & 1 & & \\
\hline High School/Equivalent & 1.31 & $1.00-1.73$ & 0.05 & 1.18 & $0.57-2.46$ & 0.65 \\
\hline Above High School & 1.18 & $0.80-1.75$ & 0.40 & 0.70 & $0.31-1.60$ & 0.39 \\
\hline \multicolumn{7}{|l|}{ Income Level } \\
\hline Low $^{a}$ & 1 & & & 1 & & \\
\hline Middle & 0.85 & $0.70-1.04$ & 0.11 & 1.19 & $0.69-2.04$ & 0.53 \\
\hline High & 0.78 & $0.55-1.11$ & 0.16 & 1.52 & $0.76-3.04$ & 0.23 \\
\hline \multicolumn{7}{|l|}{ Occupation } \\
\hline Blue Collar, Semi-Routine ${ }^{a}$ & 1 & & & 1 & & \\
\hline White Collar and Professional & 0.89 & $0.59-1.32$ & 0.55 & 0.75 & $0.43-1.31$ & 0.30 \\
\hline White Collar, Semi-Routine & 0.93 & $0.60-1.45$ & 0.75 & 1.10 & $0.62-1.94$ & 0.73 \\
\hline Blue Collar, High Skill & 1.03 & $0.67-1.59$ & 0.89 & 1.30 & $0.87-1.95$ & 0.20 \\
\hline Not Working & 1.31 & $0.76-2.25$ & 0.32 & 0.94 & $0.28-3.14$ & 0.91 \\
\hline \multicolumn{7}{|l|}{ Body Mass Index $\left(\mathrm{kg} / \mathrm{m}^{2}\right)$} \\
\hline Underweight $^{a}$ & 1 & & & 1 & & \\
\hline Normal & 1.36 & $0.71-2.59$ & 0.35 & 1.84 & $0.37-9.25$ & 0.44 \\
\hline Overweight & 1.08 & $0.60-1.94$ & 0.80 & 2.07 & $0.45-9.51$ & 0.34 \\
\hline Obese & 1.63 & $0.86-3.11$ & 0.13 & 2.84 & $0.58-14.04$ & 0.19 \\
\hline \multicolumn{7}{|l|}{ Smoking } \\
\hline Never $^{a}$ & 1 & & & 1 & & \\
\hline Former & 1.69 & $1.16-2.45$ & 0.01 & 2.24 & $1.32-3.80$ & 0.004 \\
\hline Current & 3.87 & $2.87-5.23$ & $<0.001$ & 7.17 & $4.23-12.16$ & $<0.001$ \\
\hline \multicolumn{7}{|l|}{ Physical Activity } \\
\hline About the Same ${ }^{a}$ & 1 & & & 1 & & \\
\hline Less Active & 1.15 & $0.85-1.55$ & 0.35 & 1.19 & $0.60-2.35$ & 0.61 \\
\hline More Active & 0.69 & $0.55-0.85$ & 0.001 & 0.90 & $0.47-1.70$ & 0.73 \\
\hline
\end{tabular}

${ }^{a}$ Reference group

${ }^{b}$ Physical activity compared to others of same age

demographics, health status, and behavioral characteristics [31]. In this current report of over 10,000 participants, we also found that lower income correlated with higher CRP levels. In contrast, Yang et al. found that high SEP was associated with increased levels of inflammation and obesity in African-American males [14]. However, their study was focused on young adults. Our analysis incorporated both CRP and fibrinogen and notably adjusted for age, race, gender, BMI, education, occupation, income, and behavioral factors including physical activity and smoking.

The association between lower income and higher CRP levels, but lack of association with fibrinogen levels, may be explained by CRP levels being a more accurate 
Table 5 Multivariate Analysis - Cancer-specific Mortality Adjusted for Inflammatory Markers and Other Factors, Stratified by Income Level

\begin{tabular}{|c|c|c|c|c|c|c|}
\hline \multirow[t]{2}{*}{ Factors } & \multicolumn{3}{|c|}{ NHANES III 1988-1994 } & \multicolumn{3}{|c|}{ NHANES 1999-2002 } \\
\hline & Hazard Ratio & 95\% Confidence Interval & $P$-value & Hazard Ratio & 95\% Confidence Interval & $P$-value \\
\hline \multicolumn{7}{|l|}{ Low Income } \\
\hline \multicolumn{7}{|l|}{ C-Reactive Protein ${ }^{a}$} \\
\hline$<0.22 \mathrm{mg} / \mathrm{dL}$ & 1 & & & 1 & & \\
\hline $0.22-0.99 \mathrm{mg} / \mathrm{dL}$ & 1.23 & $0.88-1.74$ & 0.22 & 0.77 & $0.35-1.67$ & 0.49 \\
\hline$>0.99 \mathrm{mg} / \mathrm{dL}$ & 1.83 & $1.10-3.04$ & 0.02 & 1.46 & $0.87-2.45$ & 0.15 \\
\hline Fibrinogen ${ }^{a}$ & 1.001 & $1.000-1.003$ & 0.10 & 0.997 & $0.994-1.000$ & 0.08 \\
\hline \multicolumn{7}{|l|}{ Middle Income } \\
\hline \multicolumn{7}{|l|}{ C-Reactive Protein ${ }^{a}$} \\
\hline$<0.22 \mathrm{mg} / \mathrm{dL}$ & 1 & & & 1 & & \\
\hline $0.22-0.99 \mathrm{mg} / \mathrm{dL}$ & 0.87 & $0.63-1.19$ & 0.37 & 1.05 & $0.39-2.82$ & 0.92 \\
\hline$>0.99 \mathrm{mg} / \mathrm{dL}$ & 1.12 & $0.64-1.97$ & 0.69 & 0.61 & $0.17-2.11$ & 0.42 \\
\hline Fibrinogen $^{a}$ & 1.000 & $0.998-1.002$ & 0.67 & 1.001 & $0.998-1.01$ & 0.43 \\
\hline \multicolumn{7}{|l|}{ High Income } \\
\hline \multicolumn{7}{|l|}{ C-Reactive Protein ${ }^{a}$} \\
\hline$<0.22 \mathrm{mg} / \mathrm{dL}$ & 1 & & & 1 & & \\
\hline $0.22-0.99 \mathrm{mg} / \mathrm{dL}$ & 0.84 & $0.44-1.63$ & 0.60 & 1.07 & $0.53-2.18$ & 0.84 \\
\hline$>0.99 \mathrm{mg} / \mathrm{dL}$ & 1.41 & $0.62-3.19$ & 0.41 & 2.87 & $0.88-9.37$ & 0.08 \\
\hline Fibrinogen $^{a}$ & 1.000 & $0.997-1.003$ & 0.94 & 1.002 & $0.998-1.01$ & 0.36 \\
\hline
\end{tabular}

${ }^{a}$ Adjusted for gender, race, education, occupation, body mass index, smoking status, and physical activity

representation of lifetime inflammation. According to Davillas et al., fibrinogen levels vary by other physiological functions of the body [32]. Due to our efforts to minimize confounding, our adjusted sample may have shown a non-significant association between income and fibrinogen due to controlling for confounders that may vary by fibrinogen levels.

Previous reports showed that inflammation and cancer mortality may depend on gender. One study found a positive correlation between CRP levels and cancer mortality, but only in males [33]. In another study of female participants, there was no such correlation [34]. In this current report of over 10,000 men and women, we found that higher CRP was associated with increased cancer mortality among males. However, this association was not shown in female participants. Goyal and colleagues found a similar association between CRP levels and specifically in colorectal cancer mortality [7]. While we found this relationship for the composite of cancers, further studies are warranted to investigate the varying influence of inflammation on different cancer types adjusted for a full range of demographic and socioeconomic confounders. Our data may suggest that inflammation may have a greater impact on those with lower rather than upper SEP. However, caution is warranted for interpretation of these subgroup tests as we performed 12 pre-specified subgroup analyses. The subgroup associations that we found should be tested in another sample.

In addition to finding a correlation between lower income and higher inflammation, we also found a trend that low income was associated with higher cancer mortality, though not statistically significant. This finding supports the conclusions of numerous studies. In an international systematic review, Manser and Bauerfeind found that low SEP colorectal cancer patients had higher mortality [35]. Bristow's group studied ovarian cancer patients and revealed that black people and women of lower SEP had higher mortality rates compared to white and wealthier women [36]. Our results indicate that the socioeconomic disparities found by these studies were consistent for the composite of all cancers studied in our cohort. It is possible that lower income patients have higher mortality because of poor health conditions in addition to limited access to health care [37].

Lower income participants may have higher inflammation due to higher BMI, decreased physical activity, smoking, and other life stressors altering their inflammatory response $[38,39]$. One possible mechanism for the association between inflammation and cancer mortality is the presence of the cyclooxygenase- 2 and nuclear factor kappa-B genes, which directly correlates 


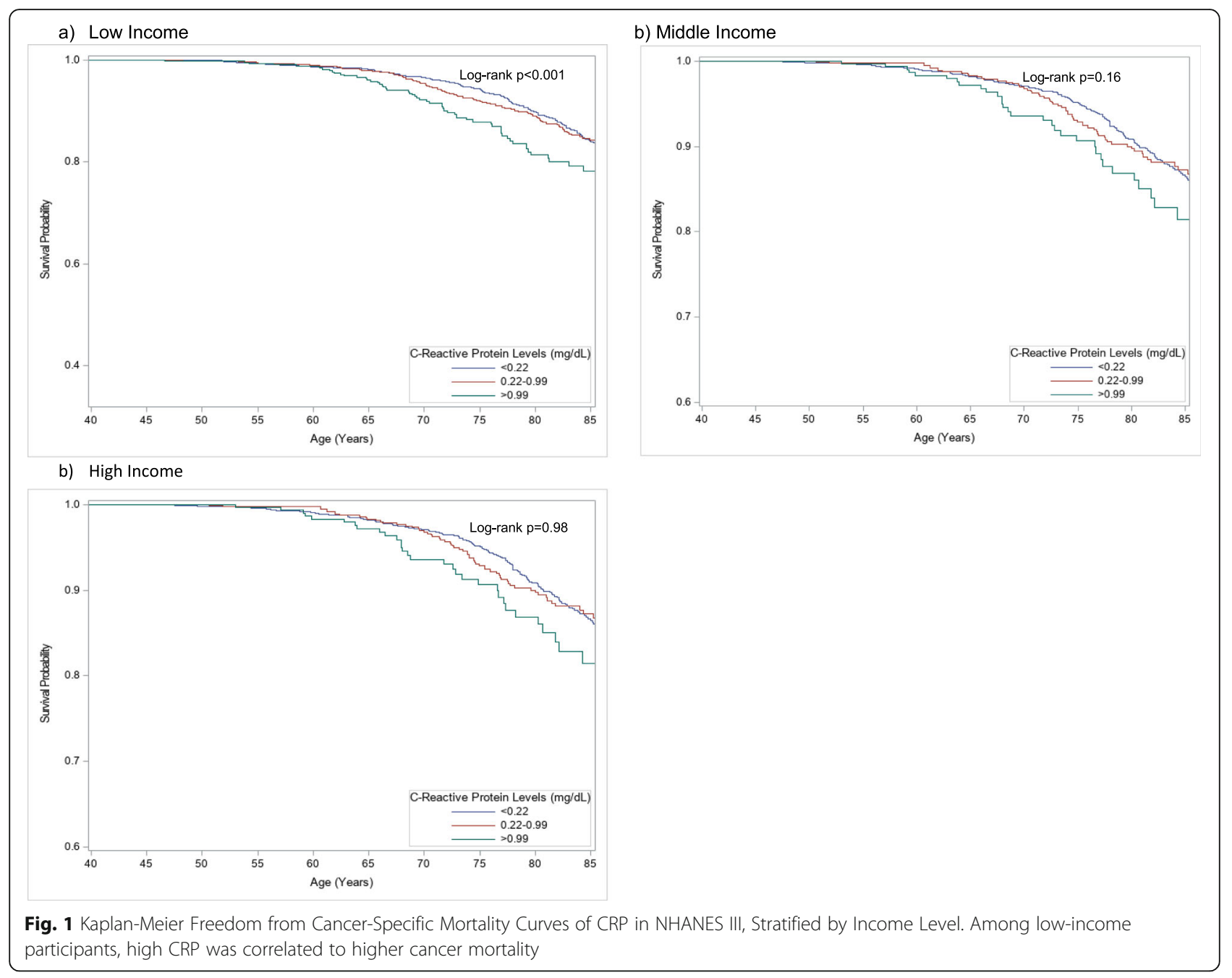

inflammation and cancer [40]. Another biologic rationale is the presence of tumor necrosis factor-alpha and interleukin-6 induced signaling, which can promote tumor growth [40]. In addition, cancers arising from infected regions of the body due to behavioral factors can cause chronic inflammation, further compromising patients' outcomes. For example, there is a strong connection between gastrointestinal infection, inflammatory bowel diseases and colorectal cancer [1, 41, 42]. Numerous trials and meta-analyses have explored the use of anti-inflammatory agents, including aspirin, in the prevention of cancer and in the reduction of metastasis in patients with cancer [43]. The benefit of antiinflammatory drugs appears to be cancer site specific, with strong benefits noted for colon, breast, and ovarian cancers [44-46]. However, our data suggest that it may be more prudent to target the use of the antiinflammatory therapies on those of lower income and/or with elevated baseline immune biomarkers, adjusted by cancer site $[47,48]$.

\section{Strengths and limitations}

Our study has limitations that may impact how generalizable these results are to all populations. Our cohort only included adults over 40 years and lacked information on specific cancer type, time of diagnosis, stage of disease, surgical and chemotherapy treatment. Without this data, we were unable to perform specific analyses on different cancer types with varying immune and inflammatory responses. In addition, NHANES 19992002 had 2344 participants in its sample compared to 7817 in NHANES III. We thus had substantially less power in the more recent NHANES study. However, the trends in the NHANES 1999-2002 data were similar to the statistically significant trends in NHANES III. Additionally, we do not have detailed information on comorbidities such as autoimmune diseases and other chronic illnesses that may alter the inflammatory response. Our study also did not collect biomarker data at various time points in relation to patient's diagnosis, treatment, and follow-up. Furthermore, CRP and fibrinogen are non- 
specific acute-phase proteins and are not completely representative of low-grade inflammation. Factors such as age, diet, and genetics may cause variation in inflammatory marker levels [49]. However, the strengths of our study include that it consists of a nationally representative cohort and is a cross-discipline investigation incorporating social science, immunology, and oncology.

Previous studies that did not control for SEP may be confounded. For example, Swede et al. observed that higher baseline CRP was correlated to higher colorectal cancer mortality; however, their findings were not adjusted for SEP [26]. Similarly, Wulaningsih et al. concluded that higher CRP was associated with a greater risk of dying from cancer after only adjusting for BMI and waist circumference [33]. However, by adjusting for education, occupation, and income, we eliminated some additional residual socioeconomic confounding.

The shorter follow-up period of the more recent NHANES sample also limited our study. The temporal differences in our model may simply be due to unstable findings, or it may be due to temporal differences across the NHANES III and NHANES 1999-2002 datasets. Our results are consistent with there being temporal differences in how CRP and fibrinogen predict outcomes. Sin et al. also found a temporal relationship between systemic inflammation and chronic obstructive pulmonary disease mortality, concluding that with 7 to 8 years of surveillance, baseline CRP levels were associated with higher rates of cardiovascular diseases and cancerspecific death [50]. In addition, Oikawa et al. showed that a temporal difference in CRP levels was associated with increased cancer mortality and heart failure [51]. The associations between inflammation and cancer mortality may have been statistically significant in the NHANES III because they were collected over a sevenyear period as opposed to four years in NHANES 19992002.

\section{Conclusions}

Our results suggest that lower income is associated with higher levels of CRP and fibrinogen. Once controlling for multiple measures of SEP, we found that elevated inflammation levels independently predicted cancer mortality only in low income participants within the NHANES III dataset. This suggests that there is limited benefit of these inflammatory markers for cancer prediction in most populations, and in the more recent cohort. While inflammatory markers may serve as potential targets for novel drug development [52], they do not appear to be productive for predicting cancer once socioeconomic confounding is more completely accounted for.

\section{Supplementary Information}

The online version contains supplementary material available at https://doi. org/10.1186/s12889-020-09923-8.

Additional file 1: Supplemental Table 1. Occupational Classifications in NHANES III and NHANES 1999-2002. Occupational Classification

Additional file 2: Supplemental Table 2. Demographic, Socioeconomic, and Behavioral Characteristics Associated with CRP Levels. Association analysis between CRP and factors

Additional file 3: Supplemental Table 3. Demographic, Socioeconomic, and Behavioral Characteristics Associated with Fibrinogen Levels. Association analysis between fibrinogen and factors

Additional file 4: Supplemental Table 4. Association of Cancer Mortality and Inflammatory Markers of All Participants. Cox-proportional hazard analysis of unadjusted (Model 1), demographic adjusted (Model 2), socioeconomic status adjusted (Model 3), and behavioral factors adjusted (Model 4)

Additional file 5: Supplemental Figure 1. Directed Acyclic Graph (DAG) depicting causal assumptions of inflammation and cancer mortality. Directed Acyclic Graph describes the relationship between inflammation and cancer mortality as well as potential confounders that is associated with both inflammation and cancer mortality.

Additional file 6: Supplemental Figure 2. Kaplan-Meier Survival from Cancer-Specific Mortality Curves by Income Level. Differences in survival outcomes stratified by income levels

Additional file 7: Supplemental Figure 3. Kaplan-Meier Survival from Cancer-Specific Mortality Curves by CRP Level. Differences in survival outcomes stratified by CRP levels

Additional file 8: Supplemental Figure 4. Kaplan-Meier Survival from Cancer-Specific Mortality Curves by Fibrinogen Level. Differences in survival outcomes stratified by fibrinogen levels

Additional file 9: Supplemental Figure 5. Kaplan-Meier Survival from Cancer-Specific Mortality Curves of CRP in NHANES 1999-2002, Stratified by Income Level. Differences in survival outcomes stratified by CRP and income levels

\section{Abbreviations}

CRP: C-reactive protein; SEP: Socioeconomic position; NHANES: National Health and Nutrition Examination Survey; CDC: Centers for Disease Control and Prevention; MEC: Mobile Examination Center; NDI: National Death Index; BMI: Body mass index; PIR: Poverty-income ratio; NCHS: National Center for Health Statistics; DAG: Directed acyclic graph; Cl: Confidence interval; HR: Hazard ratio

\section{Acknowledgements}

Denise Cobb Hale and The Fisher Family Fund for administrative support. These sources did not provide financial support in any way.

\section{Authors' contributions}

All authors have equally contributed in the conceptions of this project (JEC AKM, DSK, and DHR). AKM downloaded the necessary datasets from NHAN ES, as well as analyzed the data. DHR helped in supervising the project. JEC, AKM, DSK, and DHR interpreted data results, drafted the manuscript, and gave critical revision. All authors have read and approved the final manuscript.

\section{Funding}

Not applicable.

\section{Availability of data and materials}

The datasets generated and/or analyzed during the current study are publicly available from the Centers for Disease Control and Prevention (https://wwwn.cdc.gov/nchs/nhanes/Default.aspx).

Ethics approval and consent to participate Not applicable. 


\section{Consent for publication}

Not applicable.

\section{Competing interests}

Dr. David Rehkopf is an editorial board member for BMC Public Health.

\section{Author details}

'Division of Primary Care and Population Health, Department of Medicine, Stanford University School of Medicine, 1701 Page Mill Road, Room 229, Palo Alto, Stanford, CA 94304, USA. ${ }^{2}$ Division of Gynecologic Oncology, Palo Alto Medical Foundation, Sutter Research Institute, Palo Alto, CA, USA. ${ }^{3}$ Department of Radiation Oncology, Stanford University School of Medicine, Stanford, CA, USA.

Received: 12 May 2020 Accepted: 18 November 2020

Published online: 26 November 2020

\section{References}

1. Coussens LM, Werb Z. Inflammation and cancer. Nature. 2002;420:860-7.

2. Crusz SM, Balkwill FR. Inflammation and cancer: advances and new agents. Nat Rev Clin Oncol. 2015;12:584-96.

3. Minihane AM, Vinoy S, Russell WR, Baka A, Roche HM, Tuohy KM, et al. Lowgrade inflammation, diet composition and health: current research evidence and its translation. Br J Nutr. 2015;114:999-1012.

4. Liu J, Zhang Y, Lavie CJ, Tabung FK, Xu J, Hu Q, et al. Associations of Creactive protein and fibrinogen with mortality from all-causes, cardiovascular disease and cancer among U.S. adults. Prev Med. 2020: 106044.

5. Everett CJ, Wells BJ, Frithsen IL, Koopman RJ. Smoking, fibrinogen and cancer mortality. J Natl Med Assoc. 2007;99:328-33.

6. Jimenez RV, Kuznetsova V, Connelly AN, Hel Z, Szalai AJ. C-reactive protein promotes the expansion of myeloid derived cells with suppressor functions. Front Immunol. 2019;10. https://doi.org/10.3389/fimmu.2019.02183.

7. Goyal A, Terry MB, Jin Z, Siegel AB. C-reactive protein and colorectal cancer mortality in U.S. adults. Cancer Epidemiol Biomark Prev. 2014;23:1609-18.

8. Guida JL, Ahles TA, Belsky D, Campisi J, Cohen HJ, DeGregori J, et al. Measuring aging and identifying aging phenotypes in Cancer survivors. J Natl Cancer Inst. 2019;111:1245-54.

9. McEwen BS, Bulloch K. Epigenetic impact of the social and physical environment on brain and body. Metab Clin Exp. 2019;100S:153941.

10. Qiu J, Yu Y, Fu Y, Ye F, Xie X, Lu W. Preoperative plasma fibrinogen, platelet count and prognosis in epithelial ovarian cancer. J Obstet Gynaecol Res. 2012;38:651-7

11. Liu RS, Aiello AE, Mensah FK, Gasser CE, Rueb K, Cordell B, et al. Socioeconomic status in childhood and C-reactive protein in adulthood: a systematic review and meta-analysis. J Epidemiol Community Health. 2017; 71:817-26.

12. Rachet B, Ellis L, Maringe C, Chu T, Nur U, Quaresma M, et al. Socioeconomic inequalities in cancer survival in England after the NHS cancer plan. Br J Cancer. 2010;103:446-53.

13. Coleman MP, Rachet B, Woods LM, Mitry E, Riga M, Cooper N, et al. Trends and socioeconomic inequalities in cancer survival in England and Wales up to 2001. Br J Cancer. 2004;90:1367-73.

14. Yang YC, Johnson MP, Schorpp KM, Boen CE, Harris KM. Young adult risk factors for Cancer: obesity, inflammation, and Sociobehavioral mechanisms. Am J Prev Med. 2017;53:S21-9.

15. Jousilahti P, Salomaa V, Rasi V, Vahtera E, Palosuo T. Association of markers of systemic inflammation, $C$ reactive protein, serum amyloid $\mathrm{a}$, and fibrinogen, with socioeconomic status. J Epidemiol Community Health. 2003;57:730-3.

16. Sitenga JL, Aird G, Ahmed A, Walters R, Silberstein PT. Socioeconomic status and survival for patients with melanoma in the United States: an NCDB analysis. Int J Dermatol. 2018:57:1149-56.

17. Tyrrell J, Melzer D, Henley W, Galloway TS, Osborne NJ. Associations between socioeconomic status and environmental toxicant concentrations in adults in the USA: NHANES 2001-2010. Environ Int. 2013;59:328-35.

18. de Almeida Ferreira W, Camelo L, Viana MC, Giatti L, Barreto SM. Is subjective social status a summary of life-course socioeconomic position? Cad Saude Publica. 2018;34:e00024317..
19. Eronen J, von Bonsdorff M, Rantakokko M, Portegijs E, Viljanen A, Rantanen T. Socioeconomic status and life-space mobility in old age. J Aging Phys Act. 2016;24:617-23.

20. Dinwiddie GY, Zambrana RE, Doamekpor LA, Lopez L. The Impact of Educational Attainment on Observed Race/Ethnic Disparities in Inflammatory Risk in the 2001-2008 National Health and nutrition examination survey. Int J Environ Res Public Health. 2016:13. https://doi.org/ 10.3390/ijerph13010042.

21. McTiernan A, Friedenreich CM, Katzmarzyk PT, Powell KE, Macko R, Buchner $D$, et al. Physical activity in Cancer prevention and survival: a systematic review. Med Sci Sports Exerc. 2019;51:1252-61.

22. NHANES - National Health and Nutrition Examination Survey Homepage. 2020. https://www.cdc.gov/nchs/nhanes/index.htm. Accessed 7 Aug 2020.

23. Melbye H, Hvidsten D, Holm A, Nordbø SA, Brox J. The course of C-reactive protein response in untreated upper respiratory tract infection. $\mathrm{Br} J \mathrm{Gen}$ Pract. 2004;54:653-8.

24. 2009 HHS Poverty Guidelines. ASPE. 2015. https://aspe.hhs.gov/2009-hhspoverty-guidelines. Accessed 7 Aug 2020.

25. Rehkopf DH, Berkman LF, Coull B, Krieger N. The non-linear risk of mortality by income level in a healthy population: US National Health and nutrition examination survey mortality follow-up cohort, 1988-2001. BMC Public Health. 2008;8:383

26. Swede H, Hajduk AM, Sharma J, Rawal S, Rasool H, Vella AT, et al. Baseline serum C-reactive protein and death from colorectal cancer in the NHANES III cohort. Int J Cancer. 2014;134:1862-70.

27. Davalos D, Akassoglou K. Fibrinogen as a key regulator of inflammation in disease. Semin Immunopathol. 2012;34:43-62.

28. Visser M, Bouter LM, McQuillan GM, Wener MH, Harris TB. Elevated C-reactive protein levels in overweight and obese adults. JAMA. 1999;282:2131-5.

29. Korn EL, Graubard BI, Midthune D. Time-to-event analysis of longitudinal follow-up of a survey: choice of the time-scale. Am J Epidemiol. 1997;145: 72-80.

30. Nazmi A, Victora CG. Socioeconomic and racial/ethnic differentials of Creactive protein levels: a systematic review of population-based studies. BMC Public Health. 2007;7:212.

31. Friedman EM, Herd P. Income, education, and inflammation: differential associations in a national probability sample (the MIDUS study). Psychosom Med. 2010;72:290-300.

32. Davillas A, Benzeval M, Kumari M. Socio-economic inequalities in C-reactive protein and fibrinogen across the adult age span: findings from understanding society. Sci Rep. 2017;7. https://doi.org/10.1038/s41598-017-02888-6.

33. Wulaningsih W, Holmberg L, Ng T, Rohrmann S, Van Hemelrijck M. Serum leptin, C-reactive protein, and cancer mortality in the NHANES III. Cancer Med. 2016;5:120-8.

34. Gathirua-Mwangi WG, Song Y, Monahan PO, Champion VL, Zollinger TW. Associations of metabolic syndrome and C-reactive protein with mortality from total cancer, obesity-linked cancers and breast cancer among women in NHANES III. Int J Cancer. 2018;143:535-42.

35. Manser CN, Bauerfeind P. Impact of socioeconomic status on incidence, mortality, and survival of colorectal cancer patients: a systematic review. Gastrointest Endosc. 2014;80:42-60.e9.

36. Bristow RE, Powell MA, Al-Hammadi N, Chen L, Miller JP, Roland PY, et al. Disparities in ovarian cancer care quality and survival according to race and socioeconomic status. J Natl Cancer Inst. 2013;105:823-32.

37. Chetty R, Stepner M, Abraham S, Lin S, Scuderi B, Turner N, et al. The association between income and life expectancy in the United States, 20012014. JAMA. 2016;315:1750-66.

38. Chen E, Miller GE. Socioeconomic status and health: mediating and moderating factors. Annu Rev Clin Psychol. 2013;9:723-49.

39. Delgado C, Chertow GM, Kaysen GA, Dalrymple LS, Kornak J, Grimes B, et al. Associations of body mass index and body fat with markers of inflammation and nutrition among patients receiving hemodialysis. Am J Kidney Dis. 2017;70:817-25.

40. Kraus S, Arber N. Inflammation and colorectal cancer. Curr Opin Pharmacol. 2009;9:405-10.

41. Uemura N, Okamoto S, Yamamoto S, Matsumura N, Yamaguchi S, Yamakido $M$, et al. Helicobacter pylori infection and the development of gastric cancer. N Engl J Med. 2001;345:784-9.

42. de Martel C, Ferlay J, Franceschi S, Vignat J, Bray F, Forman D, et al. Global burden of cancers attributable to infections in 2008: a review and synthetic analysis. Lancet Oncol. 2012;13:607-15. 
43. Rothwell PM, Wilson M, Price JF, Belch JFF, Meade TW, Mehta Z. Effect of daily aspirin on risk of cancer metastasis: a study of incident cancers during randomised controlled trials. Lancet. 2012;379:1591-601.

44. Ye X, Fu J, Yang Y, Chen S. Dose-risk and duration-risk relationships between aspirin and colorectal Cancer: a meta-analysis of published cohort studies. PLoS One. 2013:8. https://doi.org/10.1371/journal.pone.0057578.

45. Luo T, Yan H-M, He P, Luo Y, Yang Y-F, Zheng H. Aspirin use and breast cancer risk: a meta-analysis. Breast Cancer Res Treat. 2012;131:581-7.

46. Merritt MA, Rice MS, Barnard ME, Hankinson SE, Matulonis UA, Poole EM, et al. Pre-diagnosis and post-diagnosis use of common analgesics and ovarian cancer prognosis (NHS/NHSII): a cohort study. Lancet Oncol. 2018; 19:1107-16.

47. Gann PH, Manson JE, Glynn RJ, Buring JE, Hennekens CH. Low-dose aspirin and incidence of colorectal tumors in a randomized trial. J Natl Cancer Inst. 1993:85:1220-4

48. Cook NR, Lee I-M, Gaziano JM, Gordon D, Ridker PM, Manson JE, et al. Lowdose aspirin in the primary prevention of cancer: the Women's health study: a randomized controlled trial. JAMA. 2005;294:47-55.

49. Low-grade inflammation, diet composition and health: current research evidence and its translation. https://www.ncbi.n/m.nih.gov/pmc/articles/ PMC4579563/. Accessed 8 Aug 2020.

50. Sin DD, Man SFP. Systemic inflammation and mortality in chronic obstructive pulmonary disease. Can J Physiol Pharmacol. 2007;85:141-7.

51. Oikawa T, Sakata Y, Nochioka K, Miura M, Abe R, Kasahara S, et al. Increased risk of cancer death in patients with chronic heart failure with a special reference to inflammation-a report from the CHART-2 study. Int J Cardiol. 2019;290:106-12

52. Pepys MB, Hirschfield GM, Tennent GA, Gallimore JR, Kahan MC, Bellotti V, et al. Targeting C-reactive protein for the treatment of cardiovascular disease. Nature. 2006;440:1217-21.

\section{Publisher's Note}

Springer Nature remains neutral with regard to jurisdictional claims in published maps and institutional affiliations.

Ready to submit your research? Choose BMC and benefit from:

- fast, convenient online submission

- thorough peer review by experienced researchers in your field

- rapid publication on acceptance

- support for research data, including large and complex data types

- gold Open Access which fosters wider collaboration and increased citations

- maximum visibility for your research: over $100 \mathrm{M}$ website views per year

At $\mathrm{BMC}$, research is always in progress.

Learn more biomedcentral.com/submissions 